\title{
Marking up lattice QCD configurations and ensembles
}

\section{P. Coddington}

School of Computer Science, University of Adelaide, Adelaide, 5005, Australia

\section{B. Joó}

Jefferson Lab, Newport News, VA 23606, USA

\section{M. Maynard}

EPCC, School of Physics, University of Edinburgh, Edinburgh EH9 3JZ, UK

D. Pleiter

Deutsches Elektronen-Synchrotron DESY, 15738 Zeuthen, Germany

\section{T. Yoshié ${ }^{* \dagger}$}

Center for Computational Sciences, University of Tsukuba, Tsukuba 305-8577, Japan

\section{ILDG Metadata Working Group}

QCDml is an XML-based markup language designed for sharing QCD configurations and ensembles world-wide via the International Lattice Data Grid (ILDG). Based on the latest release, we present key ingredients of the QCDml in order to provide some starting points for colleagues in this community to markup valuable configurations and submit them to the ILDG.

The XXV International Symposium on Lattice Field Theory

July 30 - August 42007

Regensburg, Germany

\footnotetext{
${ }^{*}$ Speaker.

†E-mail: yoshie@ccs.tsukuba.ac.jp
} 


\section{Introduction}

Sharing QCD configurations world-wide via the ILDG [1, 2, 3, 4] requires standards of notation and terminology for metadata of configurations such as lattice actions and sizes used in simulations. For this purpose, the Metadata Working Group (MDWG) [5] has been working [2, 6, 7] on designing an XML-based markup language QCDml [8], a set of rules for XML instance documents (IDs). IDs are stored in regional grid databases which are aggregated together to form the ILDG Metadata Catalogue (MDC). Because this service is queried by users, contributors (and hopefully users) are advised to understand QCDml.

After the first working version [6] was released in 2004, the MDWG has revised the QCDml schema several times [7]. Although our design strategy and the global structure of QCDml remain unchanged, many improvements have been made to meet community requirements, some of them being incompatible with previous versions. In this article, we present key ingredients of the QCDml, based on the latest release.

\section{QCDml Structure}

QCDml defines the ensemble $X M L$ and the configuration $X M L$ whose structure is shown below. The former describes metadata common across an ensemble such as <physics> information, while the latter contains configuration specific information such as a trajectory number <update>, a tag <series> which distinguishes different runs in one ensemble, <precision> of the configuration, and an <avePlaquette> value. The <algorithm> parameters can be stored in either of the XML documents. The <management> part includes information of who and when data and metadata are submitted or modified. The <implementation> part contains information of machine and code used in simulations.

The two XML IDs and the configuration itself are linked together in the following way. The Markov Chain URI (MCU) in <markovChainURI> is an unique ensemble name and appears in both XML documents. The Logical File Name (LFN) in <dataLFN> of the configuration XML is an unique configuration name and is embedded in the configuration file [9]. The format of the MCU (LFN) is "mc:(lfn:)//(Name of Regional Grid)/(Regional Grid Dependent String)“".

Root elements, <markovChain> for the ensemble XML and <gaugeConfiguration> for the configuration XML, contain names of namespaces (xmlns=) and URL's of schema (schemaLocation). A two digit version number of the QCDml (currently 1.4 for ensemble and 1.3 for configuration) is appended at the end of the namespace, and more one digit release number is added to the schemata file to identify backward compatible updates.

\section{$\underline{\text { ensemble } X M L}$}

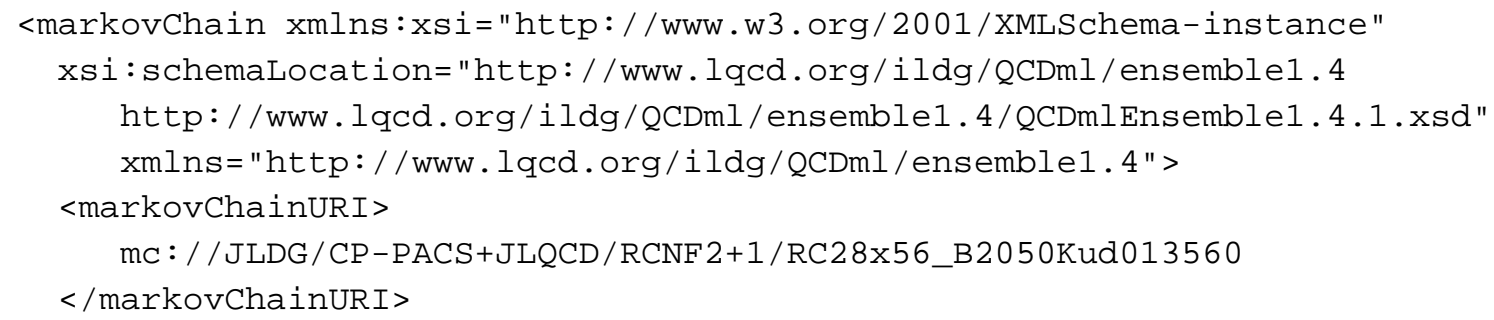




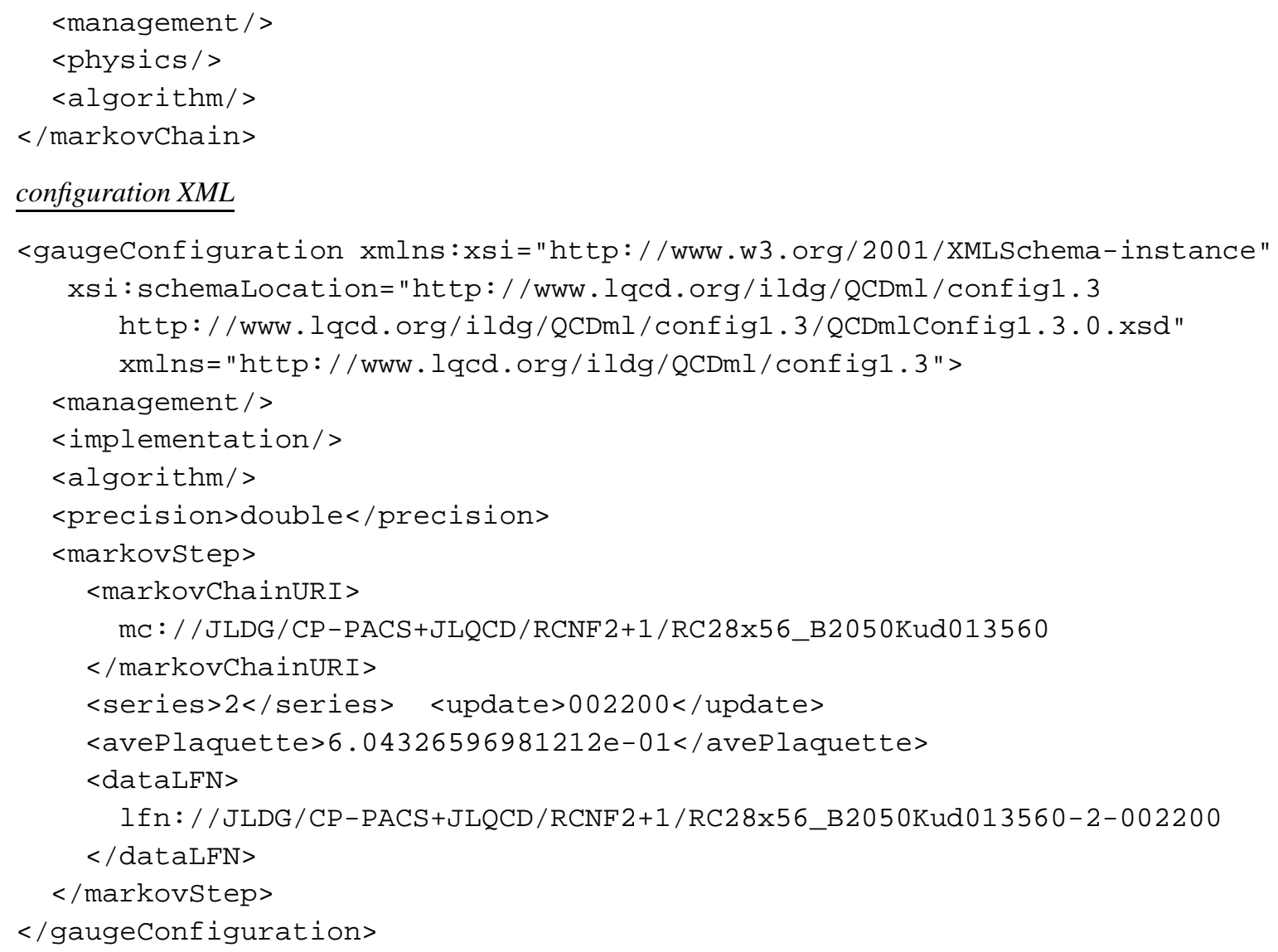

\section{Physics Part}

\subsection{Structure}

The <physics> part of the ensemble XML consists of <size>, <action> and optional <observables $\rangle$. The $\langle$ size $>$ section looks like $<$ size $><$ elem $>\langle$ name $>\mathrm{X}</$ name $><$ length $>16</$ length $>$ $<$ /elem> (lengths in Y,Z,T directions) </size>

The <action> section is a central part of the ensemble XML. The example below illustrates the structure:

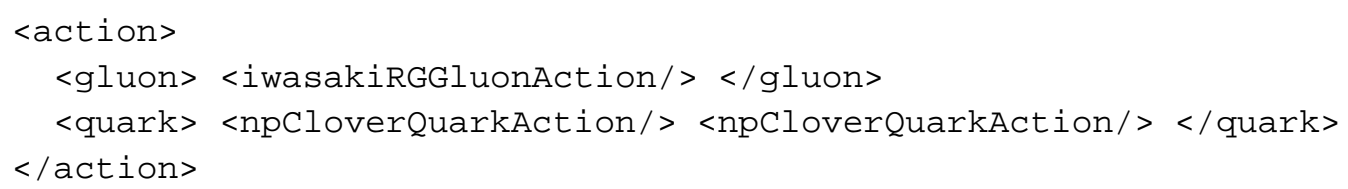

Each lattice action is described in an element block (e.g. <iwasakiRGGluonAction/>) whose name is easily understood. This strategy is taken, firstly to assign an unique name to each action, and secondly to realize a hierarchal structure of action trees in the schemata. Note that we repeat quark action blocks if coupling parameters are different, e.g. for the $N_{f}=2+1$ case. Fig. 1 shows a typical quark action. An action block consists of

- <glossary>, the URL of a document prepared by contributors using either text, pdf, ps, tex, or xml formats. The document describes full details of the action. 


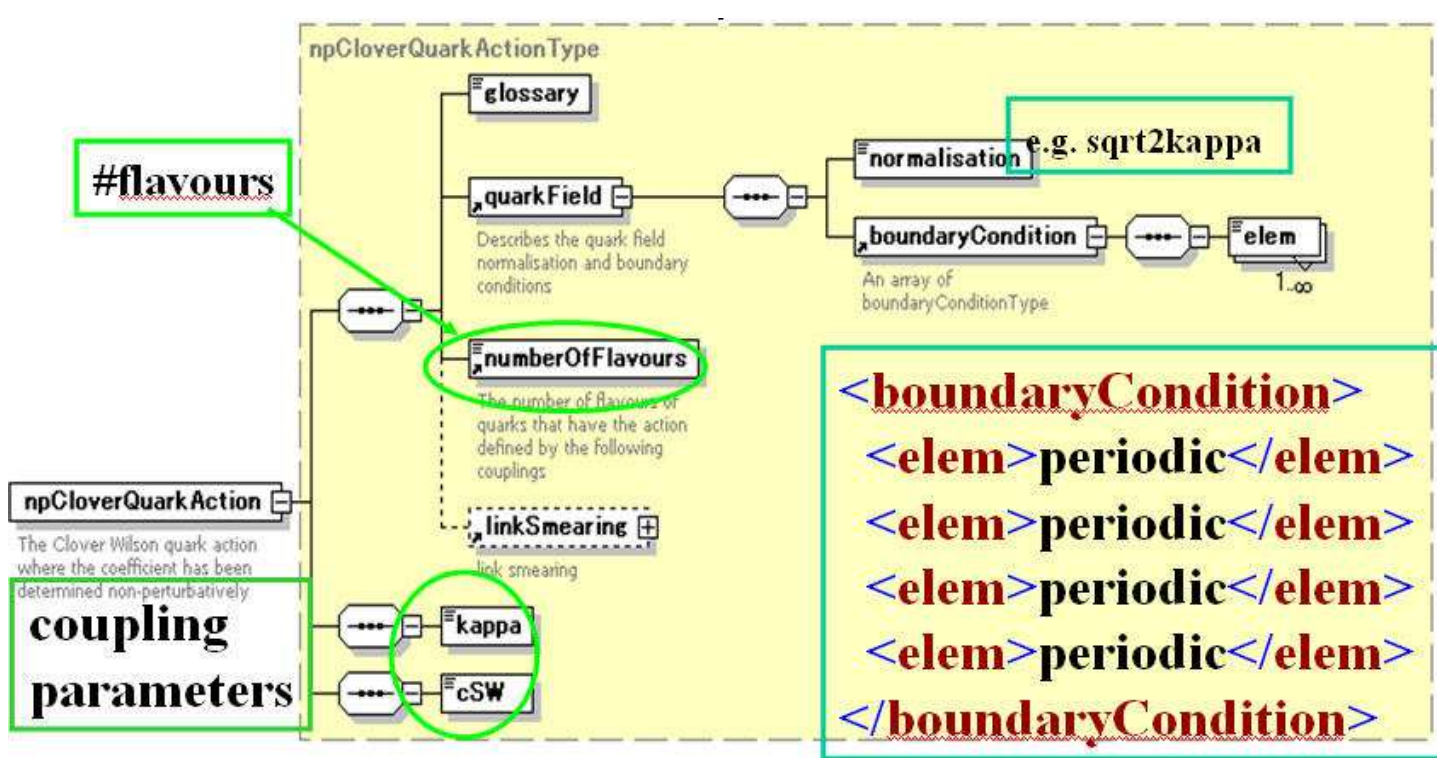

Figure 1: A graphical representation of the XML schemata for the clover quark action.

- gluon/quark field information, <gaugeField > or <quarkField>, which includes <boundaryCondition> and supplemental information such as <representation> and <normalisation>.

- <numberOfFlavours> for quark actions.

- optional <linkSmearing> block which is explained below.

- list of coupling parameters together with tadpole factors for tadpole improved actions.

The last optional block <observables> of the <physics> part displays values of measured physical quantities. Each element of the list has the following structure: <elem $>\langle$ name/> <value/> $<$ err/> <glossary/> </elem>. Currently, <name> of <observables> is one of ampi $\left(a m_{\pi}\right)$, amrho $\left(a m_{\rho}\right)$, mpi_mrho $\left(m_{\pi} / m_{\rho}\right)$, ar0 $\left(a r_{0}\right)$ and $\operatorname{arl}\left(a r_{1}\right)$. QCDml supports only dimensionless quantities, because dimensional quantities (e.g. lattice spacing) cannot be regarded as properties of an ensemble.

\subsection{Details of actions}

The current version of QCDml supports the following lattice actions (lower camel case convention).

- gluon actions: plaquetteGuonAction, iwasakiRGGluonAction, DBW2GluonAction, LuscherWeiszGluonAction, treeLevelSymanzikGluonAction, tpLuscherWeiszGluonAction, anisotropicWilsonGluonAction, anisotropicTpWilsonGluonAction

- quark actions: KSquarkAction, asqTadQuarkAction, wilsonQuarkAction, cloverQuarkAction, tpCloverQuarkAction, npCloverQuarkAction, wilsonTMQuarkAction, domainWallQuarkAction, fatLinkIrrelevantCloverQuarkAction, anisotropicWilsonQuarkAction, anisotropicCloverQuarkAction 


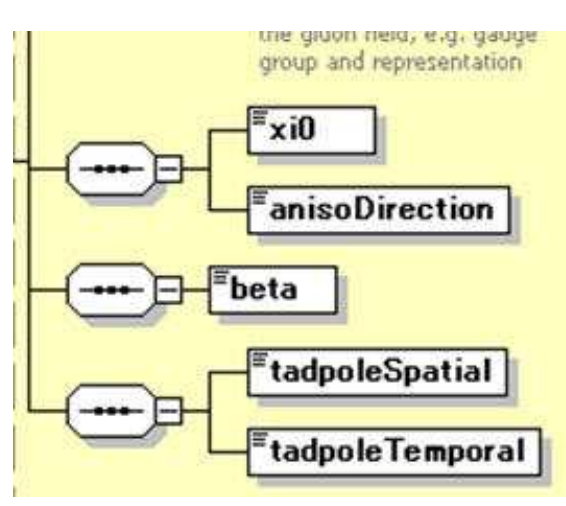

Figure 2: Coupling parameters of the anisotropic tadpole improved Wilson gluon action. <xi0> is the bare gauge anisotropy and <anisoDirection> is the direction of anisotropy.

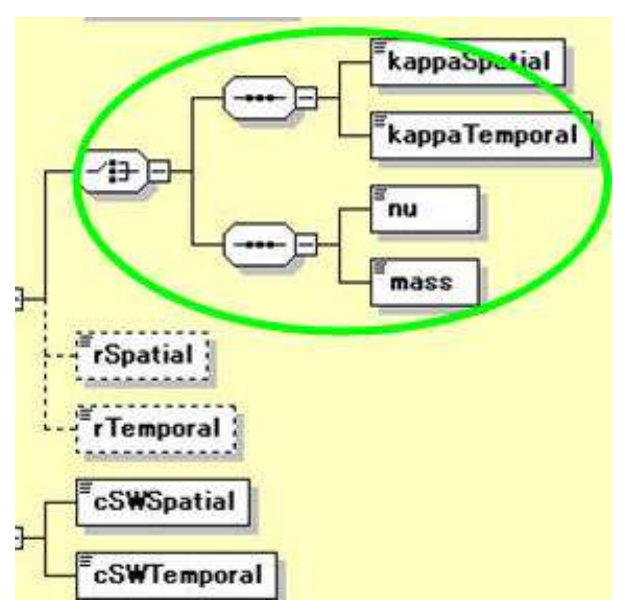

Figure 3: Coupling parameters of the anisotropic clover quark action. Wilson parameters are optional.

A list of coupling names for each action can be found in Ref. [10].

There are currently two examples of non-unique notation in QCDml.

- Gluon actions with plaquette and six-link loops (sixLinkGluonAction), such as $<$ DBW2GluonAction>, are mathematically written as

$$
S=\beta \times\left(c_{0} \sum \text { plaq. }+c_{1} \sum \text { rect. }+c_{2} \sum \text { parallelogram }+c_{3} \sum \text { char }\right) .
$$

The QCDml supports two standard normalisation of couplings;

$$
c_{0}=1, \quad c_{0}+8 c_{1}+8 c_{2}+16 c_{3}=1 .
$$

The normalisation is marked up as a value of the <normalisation> element, c0_is_one for the former (recommended for tadpole improved actions) and cs_sum_to_one for the latter (recommended for others).

- The current version of QCDml supports some anisotropic actions. See figs. 2 and 3 for examples. Note that one can choose either $\left(\kappa_{\text {Spatial }}, \kappa_{\text {Temporal }}\right)$ or $(\mu$,mass $)$ notations for anisotropic wilson/clover quark actions.

\subsection{Link smearing}

Smearing link variables is becoming one of the standard technologies for improvement. QCDml supports marking up smearing as an optional <linkSmearing> block in all quark actions. Link smearing consists of a blocking of link variables using e.g. an APE type blocking

$$
U_{\mu}(n) \leftarrow \rho_{0} U_{\mu}(n)+\rho_{1} \sum_{v(\neq \mu)}\left[U_{v}(n) U_{\mu}(n+\hat{v}) U_{v}^{\dagger}(n+\hat{\mu})+U_{v}^{\dagger}(n-\hat{v}) U_{\mu}(n-\hat{v}) U_{v}(n-\hat{v}+\hat{\mu})\right]
$$

and a projection onto the $\mathrm{SU}(3)$ group (unitarization) using e.g. a stout procedure. One repeats the set of "blocking" and "unitarization" several times. QCDml therefore marks up link smearing procedures as 
Similarly, operations (generate, add, replace, remove) affecting a configuration (or configuration XML metadata) are recorded in the <management> part of the configuration XML. Marking up these operations is optional, except for the information when a configuration has been generated.

\section{Summary and Future Work}

At present, interoperability of the regional grids has been achieved [11] for download operations and valuable configurations have already been archived [4] in the grid. Because the ILDG is becoming a new research infrastructure for the lattice QCD community, the MDWG encourages our colleagues to mark up their ensembles and configurations using QCDml.

The description of QCDml given in this report is not complete. To study more about QCDml, please visit the ILDG MDWG web site [10] where you can find sample XML documents together with human readable documents generated from annotations embedded in the QCDml schema.

The lattice actions used in simulations are becoming more and more complicated. We plan to prepare human readable documents including mathematical expressions and reference articles in the near future. We are going to update QCDml continuously to meet community requirements.

We are grateful to all members of the ILDG Metadata Working Group, in particular to C. DeTar for helpful suggestions on the manuscript. T.Y. is partly supported by the Grant-in-Aid of the Ministry of Education (No. 18104005 ) of the Japanese Government.

\section{References}

[1] C. T. H. Davies, A. C. Irving, R. D. Kenway and C. M. Maynard [UKQCD collaboration], International Lattice Data Grid, Nucl. Phys. (Proc. Suppl.) 119 (2003) 225 [hep-lat/0209121].

[2] A. C. Irving, R .D. Kenway, C. M. Maynard and T. Yoshie, Progress in building an International Lattice Data Grid, Nucl. Phys. (Proc.Suppl.) 129 (2004) 159 [hep-lat/0309029].

[3] A. Ukawa, Status of International Lattice Data Grid - An Overview -, Nucl. Phys. (Proc. Suppl.) 140 (2005) 207 [hep-lat/0409084];

K. Jansen, Status Report on ILDG activities, PoS(LAT2006)013 [hep-lat/0609012].

[4] C. DeTar, Sharing Lattices Throughout the World: An ILDG Status Report, these proceedings.

[5] Working group members: G. Andronico (INFN), P. Coddington (Adelaide), C. DeTar (Utah), R. Edwards (JLAB), B. Joo (JLAB), C. M. Maynard (Edinburgh), D. Pleiter (NIC/DESY), J. Simone (FNAL), T. Yoshie (Tsukuba)

[6] C. M. Maynard and D. Pleiter, QCDml: First milestone for building an International Lattice Data Grid, Nucl. Phys. (Proc. Suppl.) 140 (2005) 213 [hep-lat/0409055].

[7] B. Joo and C .M. Maynard, Progress in building the International Lattice Data Grid, PoS(LAT2006)028.

[8] http://www.lqcd.org/ildg

[9] http://www-zeuthen.desy.de/pleiter/ildg/\#filefmt

[10] http://www.ccs.tsukuba.ac.jp/ILDG/

[11] D. Pleiter et al. [ILDG Middleware Working Group], Towards an interoperable International Lattice Datagrid, these proceedings. 